\section{An Agent-Based Model for the Socio-Economic Monitoring of Vis- itor Streams \\ A Study Using the Example of the Harz National Park, Germany}

Gl_Forum 2018, Issue 1

Page: 297 - 315

Full Paper

Corresponding Author:

gudrun.wallentin@sbg.ac.at

DOI: 10.1553/giscience2018_01_s297

\author{
Stefan Mohr ${ }^{1}$ and Gudrun Wallentin² \\ 'UNIGIS Austria \\ 2University of Salzburg, Austria
}

\begin{abstract}
The Harz National Park (NP) offers a well-developed path network with a total length of 813 $\mathrm{km}$, as well as a high number of starting and destination points for hikes. It was visited by about 1.7 million visitors in 2014. Due to its large extent, an accurate measurement of visitor numbers and their spatio-temporal distribution is not feasible. This paper demonstrates the possibility of simulating visitor streams around Mt. Brocken buy using the agent-based model (ABM) methodology. The GAMA v1.7 RC2 modelling environment was chosen, because it has very extensive spatial operators and simulation tasks, combined with an easyto-understand modelling language. To reduce the simulation effort, a model reduction factor MRF = 10 was tested successfully: no significant change to the model was required. After an initial parameterization, a sensitivity analysis was conducted, with the results included in the final calibration. The observed error value was significantly reduced, from $R M S E_{\text {param }}=0,3817$ to $R M S E_{\text {calib }}=0,1069$, and therefore the model was applied to the study area. For the final validation, visitor numbers from other, independent investigations were used. In addition to the 12 main routes and 7 hotspots identified, basic socioeconomic indicators emerged. Hikers' changes in behaviour according to framework conditions were analysed, demonstrating the impact of current measures on the reduction of path use. The final result is a flexible and expandable baseline model, which provides a realistic picture of the spatial distribution of hikers in the study area and additional key socio-economic figures.
\end{abstract}

\title{
Keywords:
}

agent-based model, Harz National Park, socio-economic monitoring, streams of visitors, groups of hikers

\section{Introduction}

The Harz National Park covers an area of 24,732 ha (Schliebitz, 2013), is located in a typical German mountain landscape south of Hanover, and offers a well-developed hiking trail network of $813 \mathrm{~km}$, with a large number of starting and finishing points (Nationalpark Harz, 
2011a; Nationalpark Harz, 2011b). Manual counts show approximately 1.7 million visitors in 2014 (Job, Woltering, Schamel, \& Merlin, 2014). In-depth knowledge of visitors is of great importance for National Park (NP) management (Prószyńska-Bordas, 2017), since there is a conflict between the two goals of nature conservation ('protecting nature') and tourism ('allowing tourism'). It is therefore important, for future decision-making, to understand the complexity of the networked system. The study of the interrelations between man and nature, or socio-economic monitoring, sheds light on the opportunities and deficits of Harz NP and forms the basis for optimal development management.

O'Connor, Zerger, and Itami (2005) apply an agent-based model (ABM) to monitor park visitors at the Twelve Apostles National Park, Australia, and to identify the choice of trails and length of stay of park visitors at points of interest (POIs). In the work of Baumgartner (2013), an ABM is used for the Wildnispark Zürich in order to validate the number of visitors identified by the automatic counting stations. Rupf (2014) uses an ABM to show planning options in outdoor sports for hikers and mountain bikers. So far, ABM has been used to model visitor flows for just one National Park in Germany. Also, in contrast to the studies just cited, the study area of Harz NP is much greater and the path network is more than 10 times the length of those in earlier studies. Due to the large extent of the Harz NP, it is difficult to accurately measure the number of visitors and their spatial distribution.

This research aims to demonstrate that the spatial distribution of hikers in the area around the Brocken can be simulated by means of an ABM in order to answer specific management questions: (1) description of the visitors' total spatial distribution (e.g. counters for every way segment); (2) total spatial distribution of Brocken hikers and of nature-oriented hikers, which are two notable subgroups with different goals; (3) analysis of the ongoing dismantling of trails (objective: to reduce the dissection of landscape), which is important to reach the protection goals of the NP (EUROPARK DEUTSCHLAND, 2010, SP.21).

\section{Materials and Methods}

An ABM visualizes spatio-temporal changes and entities. Mathematical-deterministic methods were excluded because they do not detect emergent phenomena (Heckbert, Baynes, \& Reeson, 2010). Hikers in a natural environment, which are represented by agents, suggest the usage of ABM (Basak, Mazumdar, \& Patheriya, 2013). According to Railsback and Grimm (2012), a typical scenario for an ABM arises when the agents and their actions are important modelling components of a simulated system. 


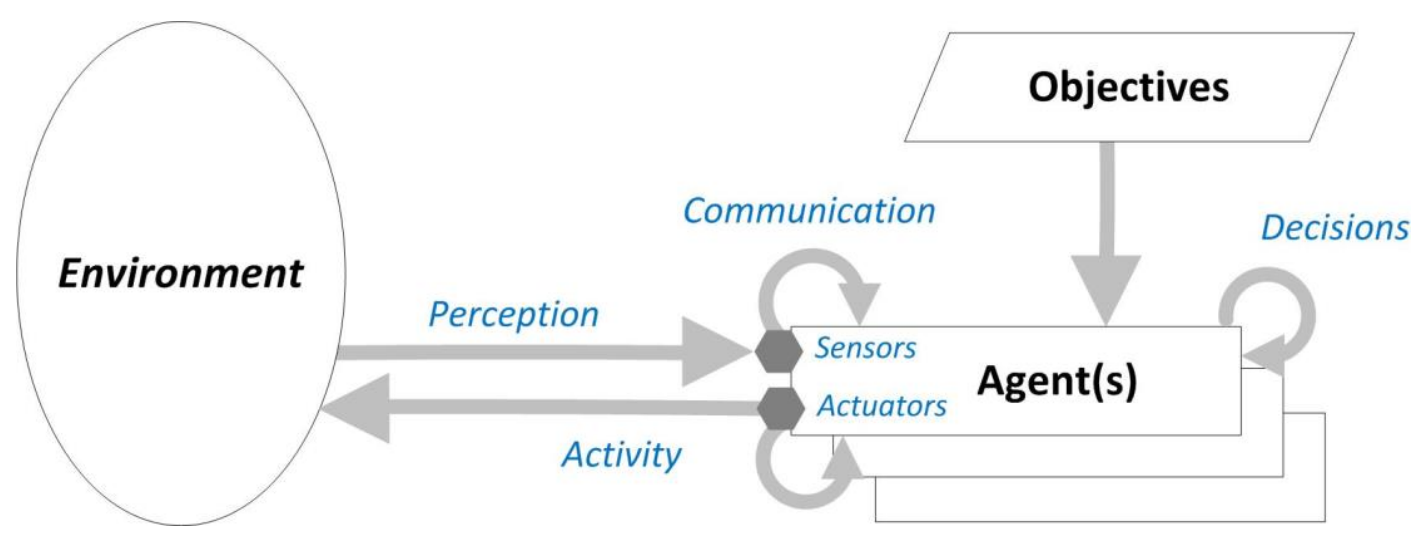

Figure 1: Interaction of an agent with its environment(according to Šalamon (2011), Nareyek (2001) and Wooldridge (2013), summarized and modified)

Šalamon (2011) and Wooldridge (2013) take the very broad, general term computer system and show how it can be used to define what we mean by an agent. According to Nareyek (2001) and Ferber (1995), agents (1) have goals, (2) perceive their environment, (3) perform actions, and (4) communicate with each other (see Figure 1).

A general approach to building a model is described by Grimm and Railsback (2013). The procedure for forming an ABM, as a multi-stage iterative process, is found in Grimm and Railsback (2013), Banos, Lang, and Marilleau (2015), and O'Sullivan and Perry (2013). Elements of all these methods are combined in the methodology for this study, as shown in Figure 2. Today, developers have access to a wide variety of ABMs from different application domains, as Abar, Theodoropoulos, Lemarinier, and O'Hare (2017) have shown in a recent paper. Because of its suitability for spatial simulations (GAMA Community, 2017), GAMA was selected to implement this model. 
Mohr \& Wallentin

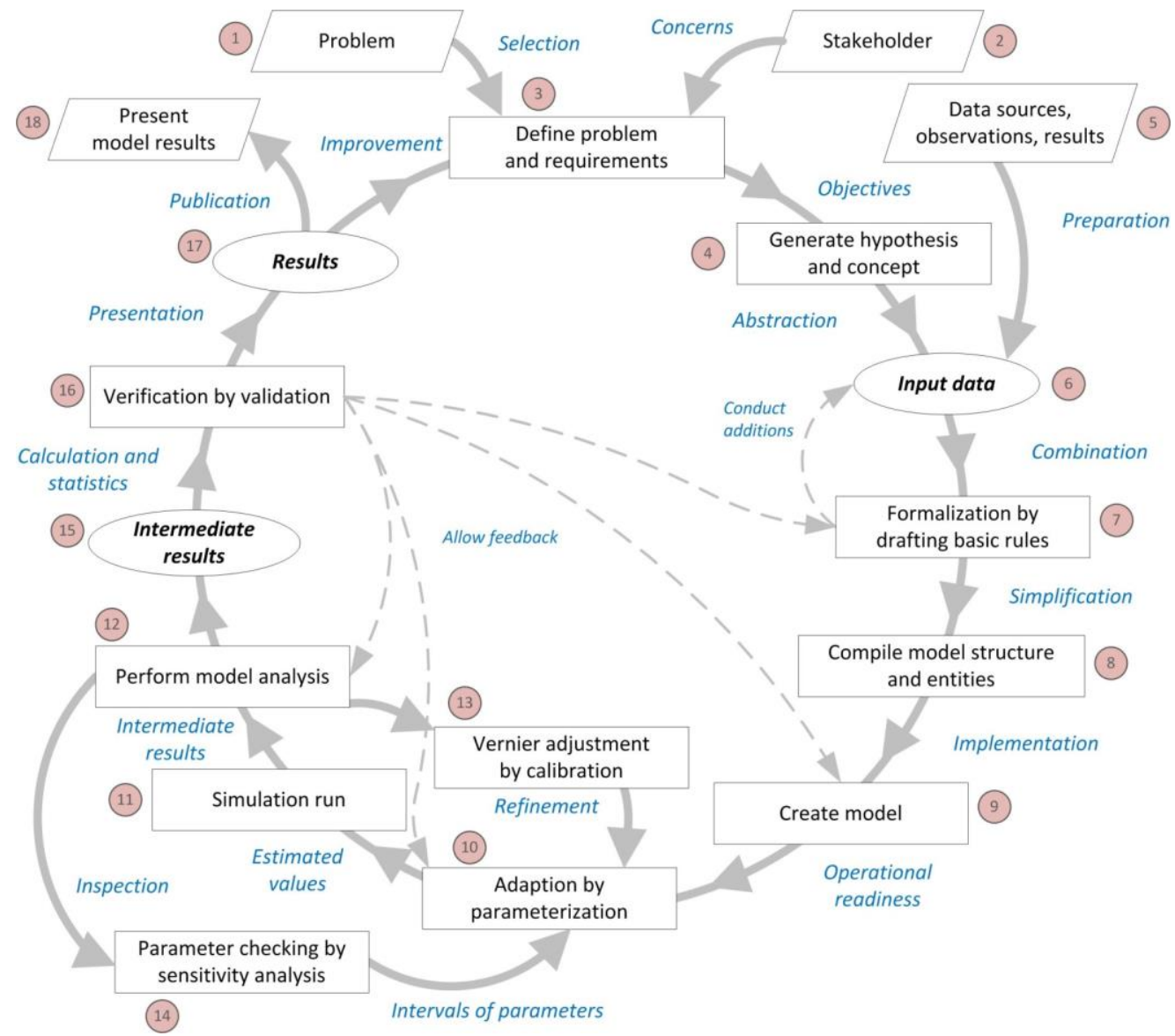

Figure 2: Combined methodology for modelling an ABM (after (modified) Anhalt and Cortez (2015), Railsback and Grimm (2012), Maki and Thompson (2017), Banos et al. (2015), and O'Sullivan and Perry (2013)) 


\section{Harz National Park}

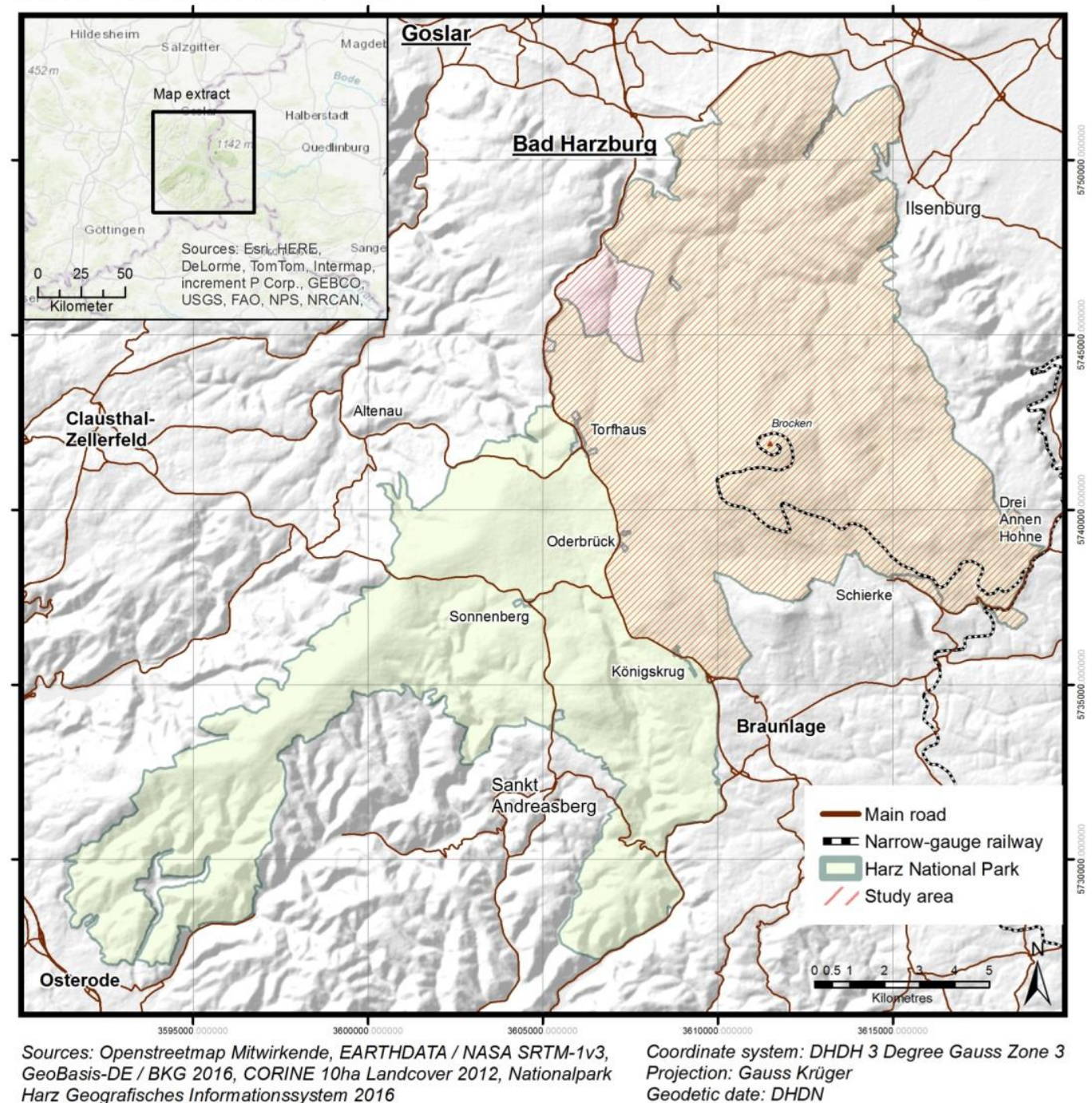

Figure 3: Delineation of the study area in the Harz NP

The Harz NP (see Figure 3) extends from the towns of Ilsenburg and Bad Harzburg in the north, the Brocken and its highlands in the centre, to Herzberg near Osterode in the south. The study area is located in the north-eastern part of Harz NP. The northern, eastern and southern edges of the study correspond to the edge of the NP, and the western and southwestern edge coincides with the federal roads B4 and B424.

According to Nationalpark Harz (2011b), the entire path network extends to around $813 \mathrm{~km}$. The attributes 'category', 'summer suitability', 'winter suitability', 'nature orientation', 'difficulty level' and the planning category were taken into account in the model. Carparks, bus stops, towns and the Brocken station of the Harz narrow gauge railways were identified as 
starting points for hikers. The total of 31 carparks is important, because most hikers will start from there. Five towns (Bad Harzburg, Torfhaus, Braunlage, Schierke and Ilsenburg) and a total of 43 bus stops were also taken into account. The 191 destinations or points of interest (POIs) in the study area can be divided into the categories 'attraction', 'viewpoint', 'information facility', 'information point', 'ranger station', 'National Park centre', 'resting place', 'hut', 'Harzer Medal for hiking' (HWN) and 'restaurant'. Due to the spatial location and significance of all places in these categories, individual attractiveness values can be assigned to them. This study considers hikers only, not mountain bikers, who are also allowed on several trails.

\section{Short ODD Protocol}

The description of the model is in a shortened form, according to the updated version of the Overview, Design concepts and Details (ODD) protocol (Grimm et al., 2010a, 2010b).

\section{Purpose}

The goal of this model is to simulate groups of hikers in the study area around the Brocken, in the Harz NP. As a result, national park management will be provided with key figures (e.g. numbers of visitors to POIs, of hikers on paths) for socio-economic monitoring.

\section{Entities, state variables and scales}

The data for the structure of the study area is in the form of shapefiles. The study area has an extent of approximately $17,850 \mathrm{~m}(\mathrm{~N}-\mathrm{S}) \times 14,650 \mathrm{~m}(\mathrm{E}-\mathrm{W})$. All distances in the shapefiles are in metres $(\mathrm{m})$; velocities are given in metres per second $(\mathrm{m} / \mathrm{s})$. Numbers are given in units, and proportional values as percentages (\%). One cycle in the model corresponds to $300 \mathrm{~s}$ real time; the simulated time period per day is 07:00 to 19:00. The length of a year is assumed to be 365 days. In the model, 14 entities are used as agents, 2 of which serve purely for representation (main roads and railway lines), 4 are designed to determine measured values (fishnet for the heat map, count points, count polygons, total count area), 1 for both counting and representing an entity, 1 represents the hikers' route network (path network), 5 serve as start and finish points (carparks, bus stops, towns, train stations and POIs), and 1 represents the groups of hikers in the model. The model uses a global reduction factor $(\mathrm{MRF}=10)$, which reduces the number of hiker-agents to be simulated. The factor was chosen so that there would be no noticeable influence on model results. A check of the exponentially increasing runtime of the model results in a value of $\mathrm{t}_{\mathrm{MRF}, 10}=8.076 \pm 668 \mathrm{~s}$ for $\mathrm{MRF} \geq 10$.

\section{Process overview and scheduling}

The structure of the model is shown in Figure 4. It is divided into the main areas of initialization, daily run and completion. The daily run is repeated until the desired simulation year has ended. 


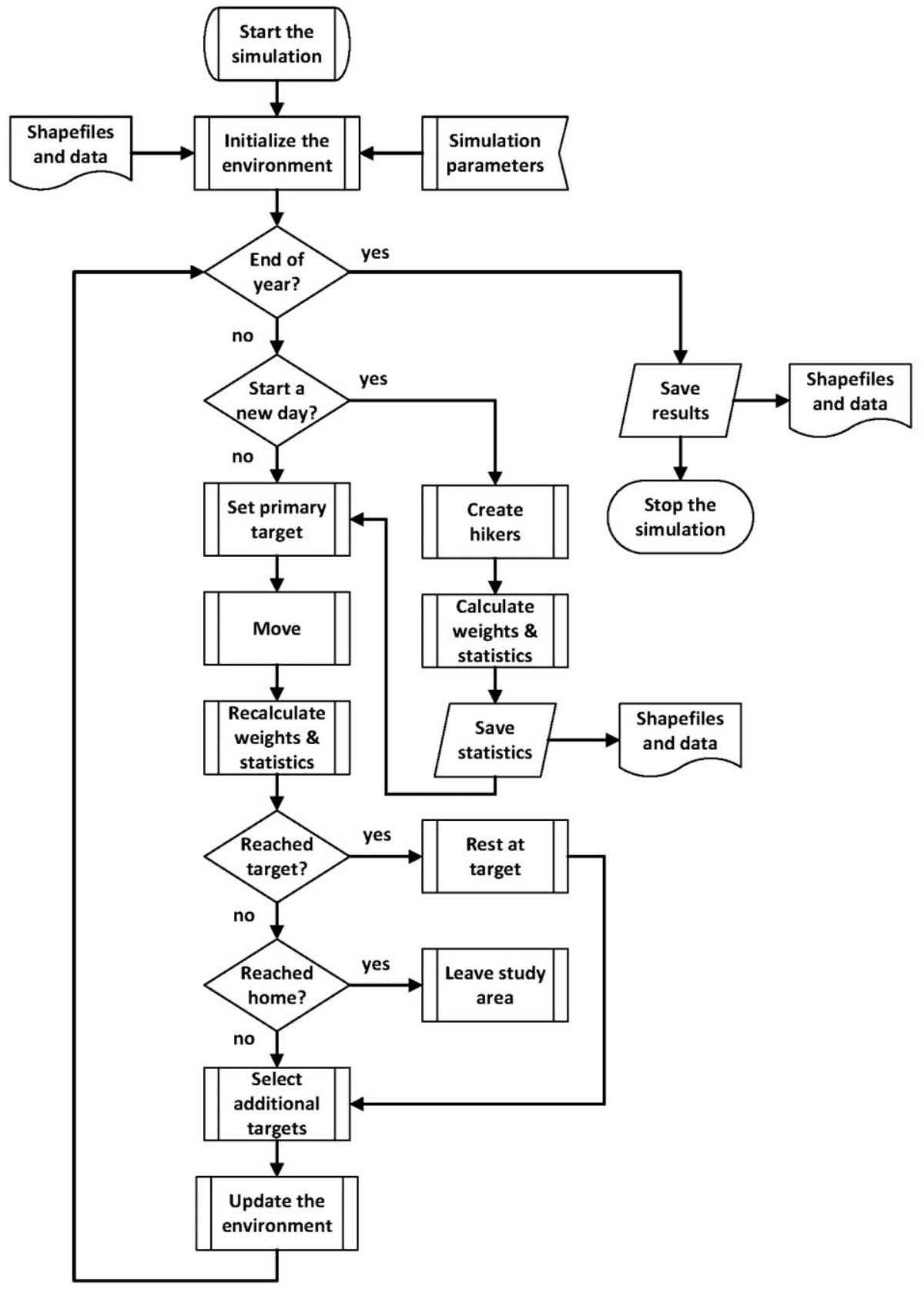

Figure 4: Flowchart of the model 


\section{Basic principles}

Figure 5 shows an Entity Relationship Diagram (ERD) to illustrate the relationships between the individual entities. Some entities serve the same purpose, so they can be grouped into scopes.

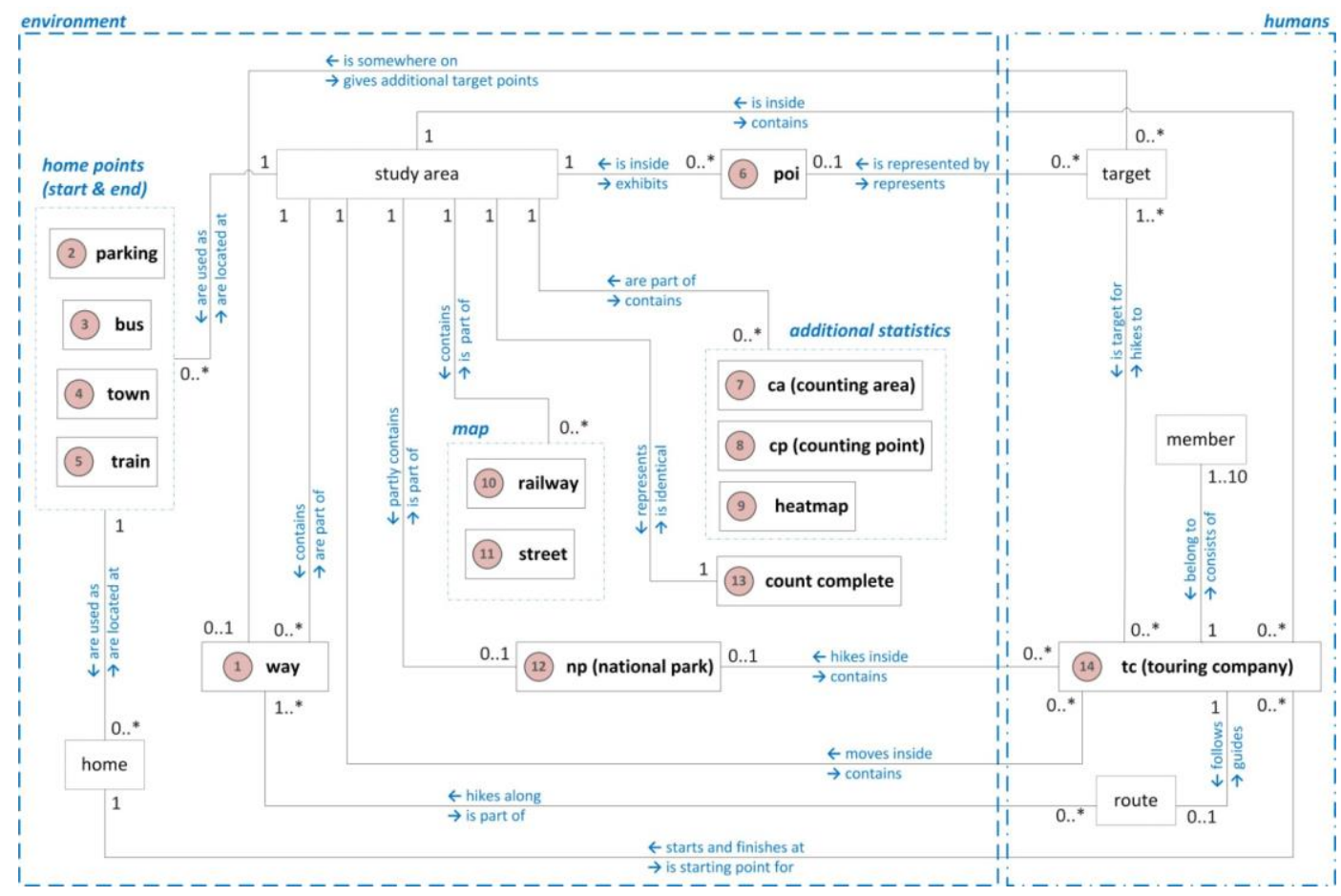

Figure 5: ERD illustrating the relationships between the entities

\section{Emergence}

The spatio-temporal distribution of the groups of hikers in the study area represents the essential emergent variable of the model. This determines the usage of the paths and the number of visitors encountered at the POIs and cannot be determined beforehand. The distribution of nature-oriented hikers is not determined deterministically, due to how they select paths.

\section{Adaption}

Parking is limited by the capacity of the carparks located in the study area. Since in most cases several carparks are located near a starting point, the groups of hikers who arrive by car select from the available carparks and thus already deviate from these starting points when choosing their carpark. The weather influences walking speed: in poor weather conditions, there is a stronger urge to reach the goals (POIs). In contrast, hikers may spend more time at the POIs in better weather conditions. Nature-oriented hikers perceive the prevailing condi- 
tions differently, preferring more natural paths and avoiding paths with higher numbers of hikers.

\section{Objectives}

Hikers in the model follow several objectives, which are also reflected in the state changes described below (see Figure 6). First, every group of hikers has to find a starting point. Then it selects a main POI within its available distance budget, which is based on a normal distribution $(\bar{x}=18 \mathrm{~km})$ and is determined individually for each hiking group. On the way to their POI and back to the starting point, a number of secondary POIs are available, which can also be accessed as destinations. Return to the starting point before nightfall is also an objective, influencing the length of the route, and taking the start time and sunset into account.

\section{Prediction, Sensing, Stochasticity and Collectives}

When adding POIs as additional targets, the hiker-agents estimate their actions using pre-set restrictions. No additional targets are added to the hike if the permissible length is exceeded or the return time would be after sunset. Hiker-agents take values from their environment and are influenced by them. These are (1) weather conditions, (2) number of hikers on trails, (3) difficulty level of trails, (4) category of trails (e.g. small paths, larger paths), and (5) the state of the trail in winter. The model contains a number of variables which exhibit a stochastic behaviour and for which a Gaussian normal distribution is assumed. Within the mod$\mathrm{el}$, there is no dynamic grouping of agents. Implicitly, the merging of hikers into groups of hikers is such an approach.

\section{Observation}

The simulation within the model includes a map showing the location of all stationary entities, the current locations of the groups of hikers, and advanced dynamic information. The simulation is equipped with 10 graph groups, which provide insights into the development of individual values within the model at runtime. In addition, a number of value monitors are available, with which the most important model results can be read. The 47 model outputs are grouped into (1) general values, (2) visitor numbers and shares, (3) visitor numbers for NP facilities, (4) visitor flows in the Brocken area, and (5) hikers who have also visited the Brocken ('Brockenhikers').

\section{Initialization}

The number of groups of hikers is set at the beginning of a new day and is based on a socalled standard number, a random element, and various seasonal factors. The number of groups to be simulated per day is approximately 876

$$
\text { standard_number_of_groups }=\frac{\text { mb_total_nlp_inside } * \mathrm{k}_{12 \text { to } 24 \mathrm{~h}}}{\overline{\mathrm{x}}_{\text {members_per_group }} * \sum_{\mathrm{i}=1}^{365} \mathrm{k}_{\text {season,i }}} * \mathrm{k}_{\text {correction }}
$$

and is broken down as follows: car $=479$, car stopover $=85$, bus $=59$, town $=32$ units, and train station $=221$ units. 


\section{Input data}

The total of 83 input variables with their starting values for the calibration of the basic model include: (1) the model controlling parameters; (2) the areas' standard numbers and factors; (3) model representation and output; (4) number of groups of hikers; (5) environment, and (6) daily information. Additional values are required to load shapefiles with fixed entities, to control the program flow, and to configure the GUI of the model. The day-dependent parameters are summarized as a CSV file and are loaded during the simulation run.

\section{Sub-models}

Hikers can adopt 7 different statuses. The status transitions are defined as in Figure 6. This sub-model (or state engine) is used for each individual travelling group. The route selection in the model is carried out by means of a Dijkstra shortest-path algorithm, which reflects the hikers' use of maps, handheld GPS and other mobile devices. The division of the overall model into additional sub-models is typical for a structured programming language.

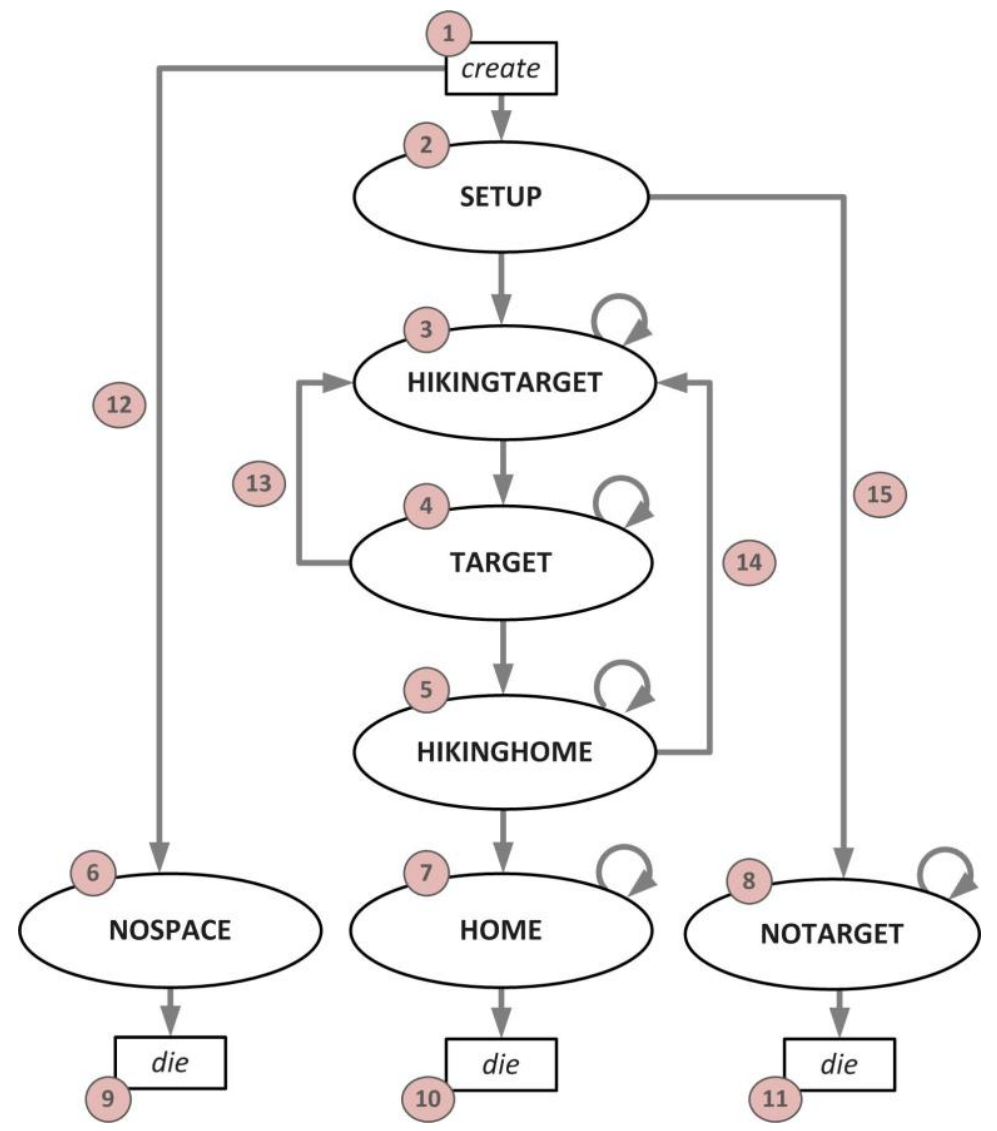

Figure 6: Sub-model of the state transitions of groups of hikers 


\section{Parameterization, sensitivity analysis, calibration and validation}

The parameterization of the model was done by assigning attraction levels to the start and finish points. For towns as starting points, their attractiveness is assumed to be proportional to their number of inhabitants. The bus stops of the two routes inside the NP receive an attractiveness of $1 / 10$ of the attractiveness of the remaining bus stops, because they have a reduced timetable compared to the bus stops on the federal roads. Bus stops at the Molkenhaus and the Lynx Enclosure are set to 10 and 40 times higher respectively because of their popularity; stops in the Torfhaus are set at 20 times higher compared with the normal bus stops. For the carparks located in the study area, the attractiveness is considered to be proportional to the usage of the carpark combined with its capacity. The attractiveness of the possible return points for hikers travelling down the Brocken is assumed to be inversely proportional to the distance travelled. For the attractiveness of the destination points, the initial estimated values shown in Table 1 are determined. These reflect a factor proportional to their estimated importance in the NP.

In the sensitivity analysis, selected values were examined for their realistic value limits and their influence on the model: (1) average tour length, (2) percentage distribution of the 3 groups of hiker types, and (3) number of winter days. The hiker density as a function of the length of the tour shows a clearly visible change in the interval of $[a ; b]=[3,000 ; 15,000] \mathrm{m}$. For a mean tour length of approximately $18,000 \mathrm{~m}$, the density distribution varies only occasionally. The percentage distribution of the different hiker-group types has only a low sensitivity in the model. For this reason, the assumed proportions of target-oriented, nature-oriented and HWN hikers are maintained at $80 \%, 15 \%$ and $5 \%$ respectively. It was shown that the number of winter days in the simulation $[a ; b]=$ $[0 ; 15]$ has little influence on the results and therefore has a low sensitivity. For this reason, the number of winter days is set at $\mathrm{n}=10$, in line with expert opinion.

Table 1: Attractions of the different POI types

\begin{tabular}{ll}
\hline \multicolumn{1}{c}{ POI-type } & \multicolumn{1}{c}{ Attractivity (basic value) } \\
\hline Touristic attractions & 100, Baumwipfelpfad: 2.000 \\
Viewpoint & 10, Brocken: 1.000, Rabenklippen: 100, Wurmberg: 100 \\
Information facility & 20, Luchsgehege: 50 \\
Ranger station with Infor- & 40 \\
mation point & 5 \\
National Park Centre & 5 \\
Ranger station only & 10 \\
Picnic area & 5, HWN 170, 169, 168, 136, 001: 10, HWN 012, 002, 009: \\
Harzer hiking medal & 20 \\
& 150, Brocken, Molkenhaus and Rabenklippe: 250 \\
Restaurant &
\end{tabular}


For the calibration, an iterative process was chosen, in which the attractiveness of the POIs was adjusted over a total of 11 runs. The determination of the quality of the individual models during the calibration was based on the percentage deviation $1-y_{i, \text { model }} / y_{i, \text { target }}$. In order to obtain an overall, model-wide, index based on Railsback and Grimm (2012), an RMSE error (used by Hedderich \& Sachs (2016) value was calculated.

$$
R M S E_{\text {Model }}=\sqrt{\frac{\sum_{i=1}^{n}\left(1-\frac{y_{i, \text { Model }}}{y_{i, \text { target }}}\right)^{2}}{n}}
$$

For the 34 result values of the baseline model, this is calculated as $R M S E_{\text {Kalib }}=0,1069$ for $\mathrm{n}=28$ simulation runs. For comparative purposes, the value of the previously parameterized but not yet calibrated model was $R M S E_{\text {Paramt }}=0,3817$, and an improved degree of suitability of the calibrated baseline model to the study area was clearly given. During calibration, the attractiveness of the starting points and POIs, and the standard start values ( $\mathrm{car}=442$, $\operatorname{car}($ stopover $)=80$, bus $=57$, city $=31$ and train station $=207)$ and number of winter days $(\mathrm{p}=0.11)$ were changed.

For the final validation of the basic model, visitor numbers from additional studies by Steingaß and Wendt (2006), Menzel, Groß, and Drexer (2013) and Groß and Menzel (2016) were available, which were not used when building the model. A closer look at the results of the 13 validation scores shows that the spread of hikers across trails was between good and very good. A comparison with the 'Goetheweg' trail provides values with a small absolute deviation of $1.10 \%$. For longer distances, the absolute deviations increase by up to $10.48 \%$. This suggests that at least one predictor in this area was not included in the model. Since the ratios of the results are consistent (and not reversed), a fundamentally plausible model can be assumed.

\section{$5 \quad$ Results}

For the whole study area, the annual sum of visitors is $1,515,464$, with a concentration of 561,702 (about 37.1\%) at the Brocken. 389,613 people (about 69.4\%) travel using the Brocken Railway, and 172,089 (about 30.6\%) hike to the Brocken. Thus, there is a spatial concentration of visitors in the area around the Brocken in the central part of the study area, which can be examined in more detail (see Figure 7). This concentration is primarily in the Scharfenstein, Torfhaus, Schierke and Hohne areas. Noticeable in this context is the area to the east of the Brocken, which receives a comparatively small number of visitors. One of the main causes is the difficulty for hikers of finding a suitable starting point, as this eastern area is furthest away from points of entry to the NP. Inspection of the visitor counts at the level of the path segments shows more differentiated results, which allows the identification of 12 main travel axes (see Figure 8 a) for the basic model. There are further zones with small numbers of visitors, in the southern and northern parts of the study area, as well as in the Bruchberg area. 


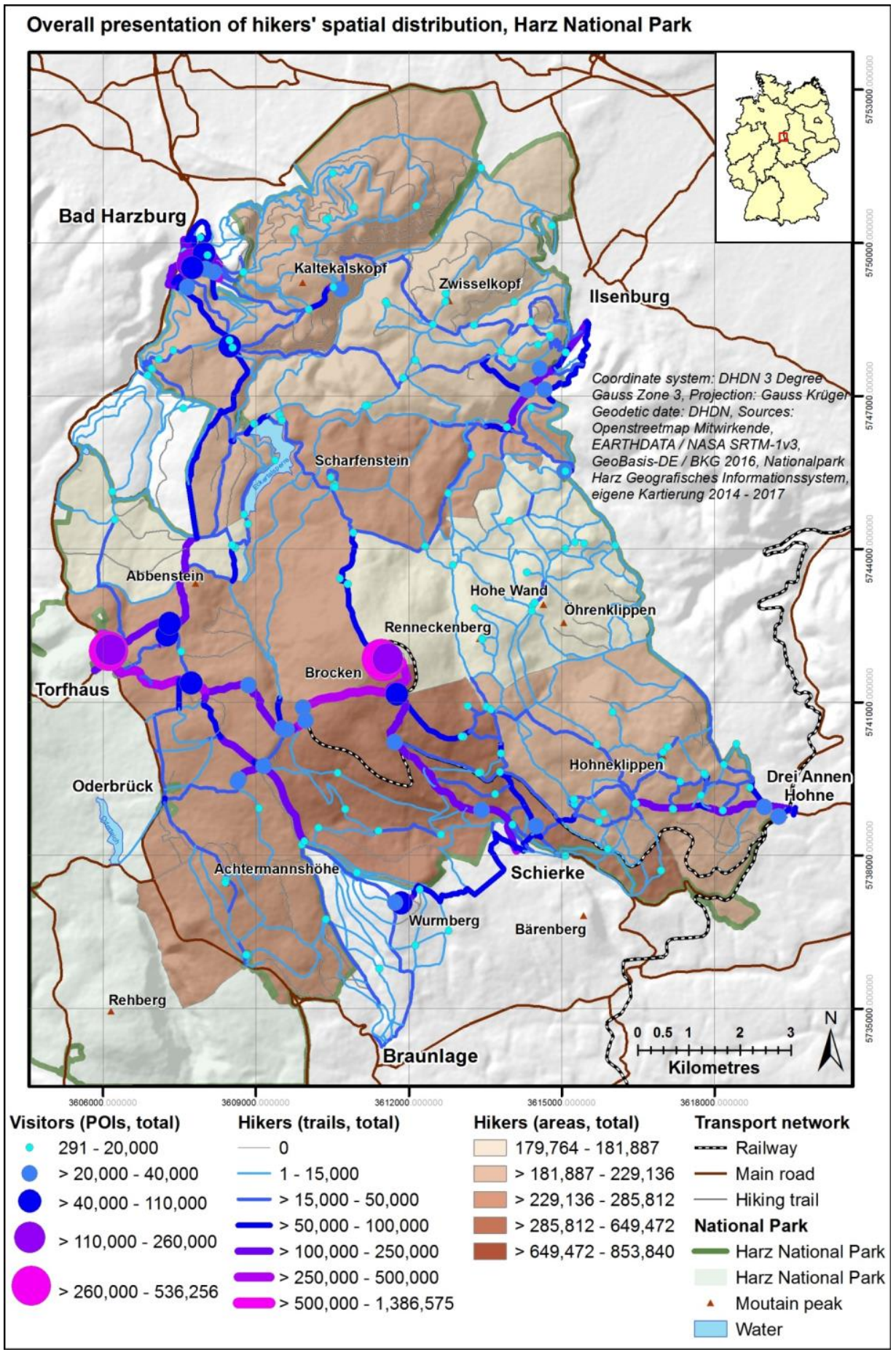

Figure 7: Overall presentation of hikers' spatial distribution (paths, POls and individual areas) 


\section{Main axes and Hotspots in the Harz National Park}

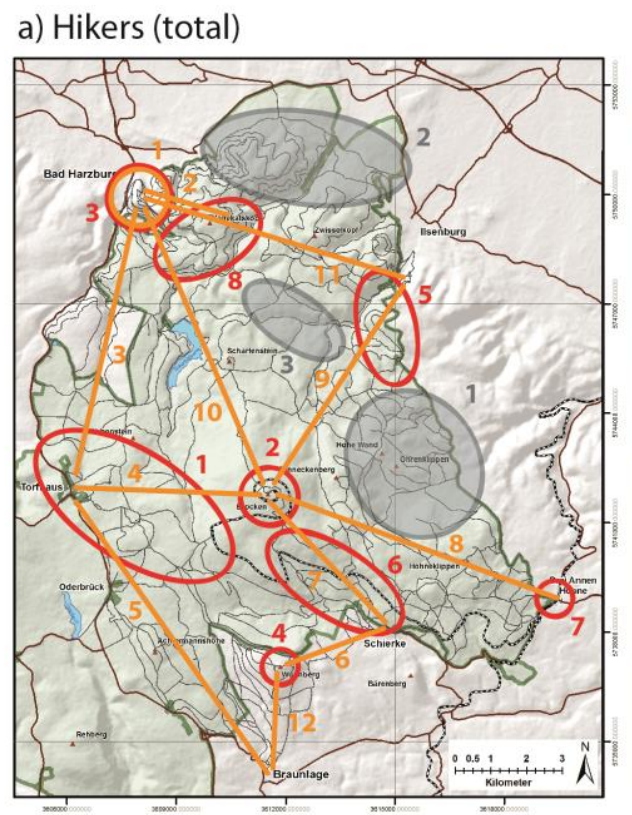

c) Brockenhikers

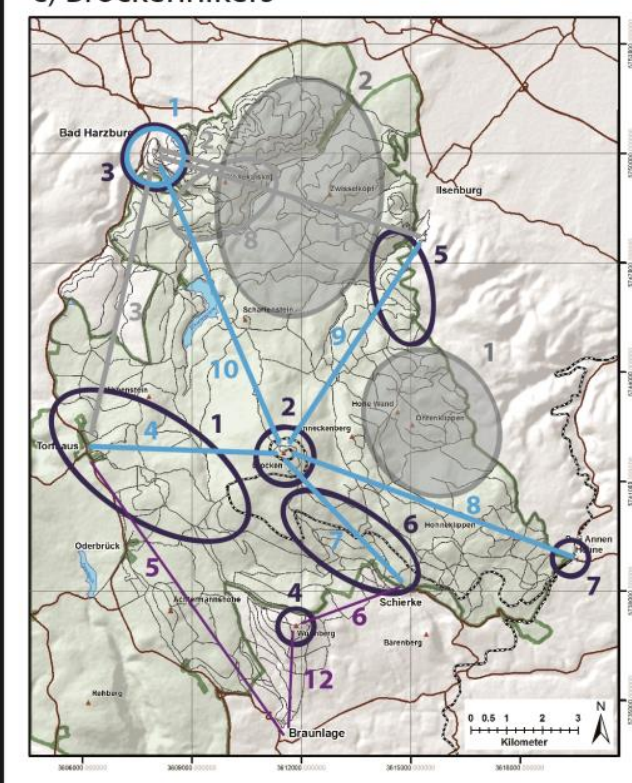

b) Nature-oriented hikers

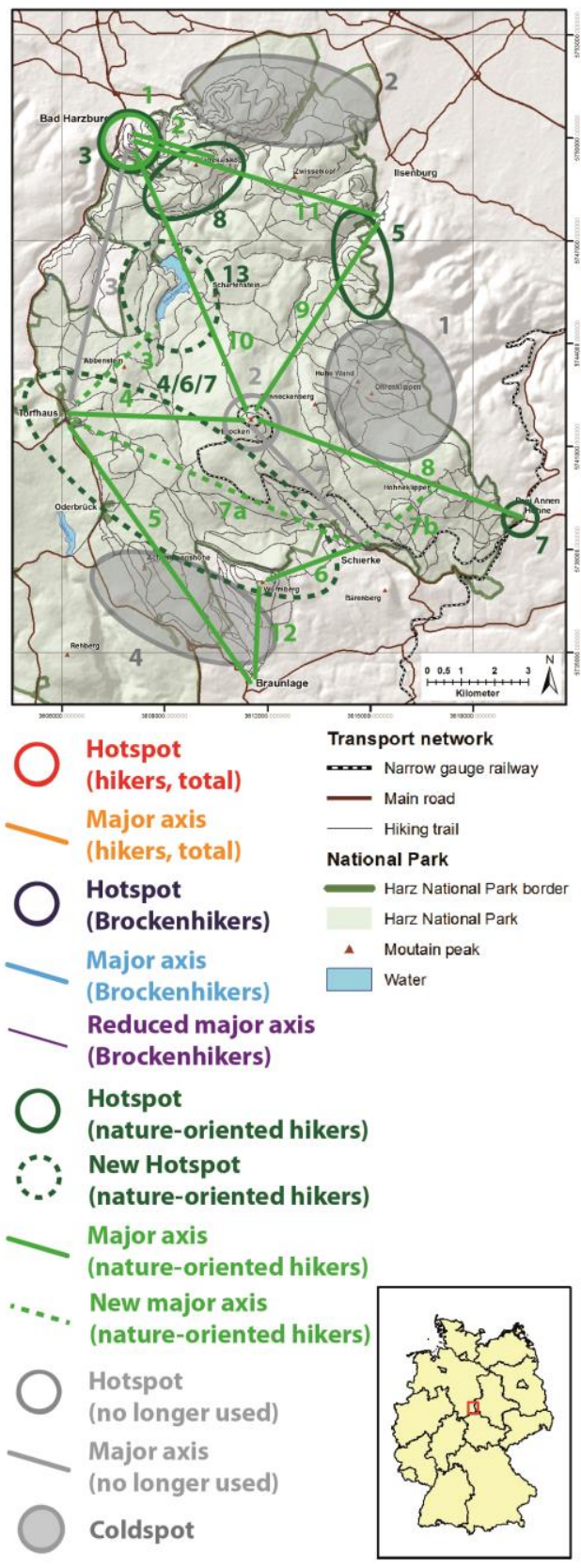

Figure 8: Schematic representation of main paths, hotspots and coldspots in the study area 
The distribution of visitors between the POIs ranges very widely, from 291 to 536,256, with an average of 21,970 people. This leads to the formation of 7 hotspots, with a clear concentration at Torfhaus $(620,729$ at the counting point) and on the Brocken plateau $(561,702)$. Further hotspots are located around hikers' primary starting areas at Schierke, Drei Annen Hohne, Ilsenburg and Bad Harzburg.

There is a wide distribution of nature-oriented hikers in the study area. At the same time, there is a shift of hikers away from the main routes to side routes or new hotspots (see Figure $8 \mathrm{~b}$ ). This leads to an increased use of the Torfhaus-Eckerstausee, Torfhaus-Wurmberg and Torfhaus-Schierke routes, and in the Plessenburg area. In contrast, the paved Brocken road between Schierke and the Brocken attracts only a small proportion of nature-oriented hikers. To bypass the Brocken road towards Hohneklippen, they use a north-eastern route. The distribution of hikers in the district of Plessenburg falls sharply, an effect which is also noticeable towards the northern and southern edges of the study area. The largest concentration of hikers is found in a corridor between Torfhaus, Schierke and Drei Annen Hohne. A significantly increased use around the Eckerstausee is found, which corresponds to the suitability of the paths for nature-oriented hikers.

The concentration of the Brockenhikers, who have the Brocken as their main target, results in a different spatial distribution from that of the group comprising all hikers. There is a concentration on 6 of the 12 main axes, with 2 that are clearly recognizable as the most-used ones (see Figure 8 c): Torfhaus-Brocken, and Schierke-Brocken. On the Brocken itself, the viewpoint and the two restaurants have high numbers of visitors (view point: 536,256 visitors; restaurants: 266,485 and 182,855), so that they also belong to the group of the 10 most visited POIs in the Harz NP. 


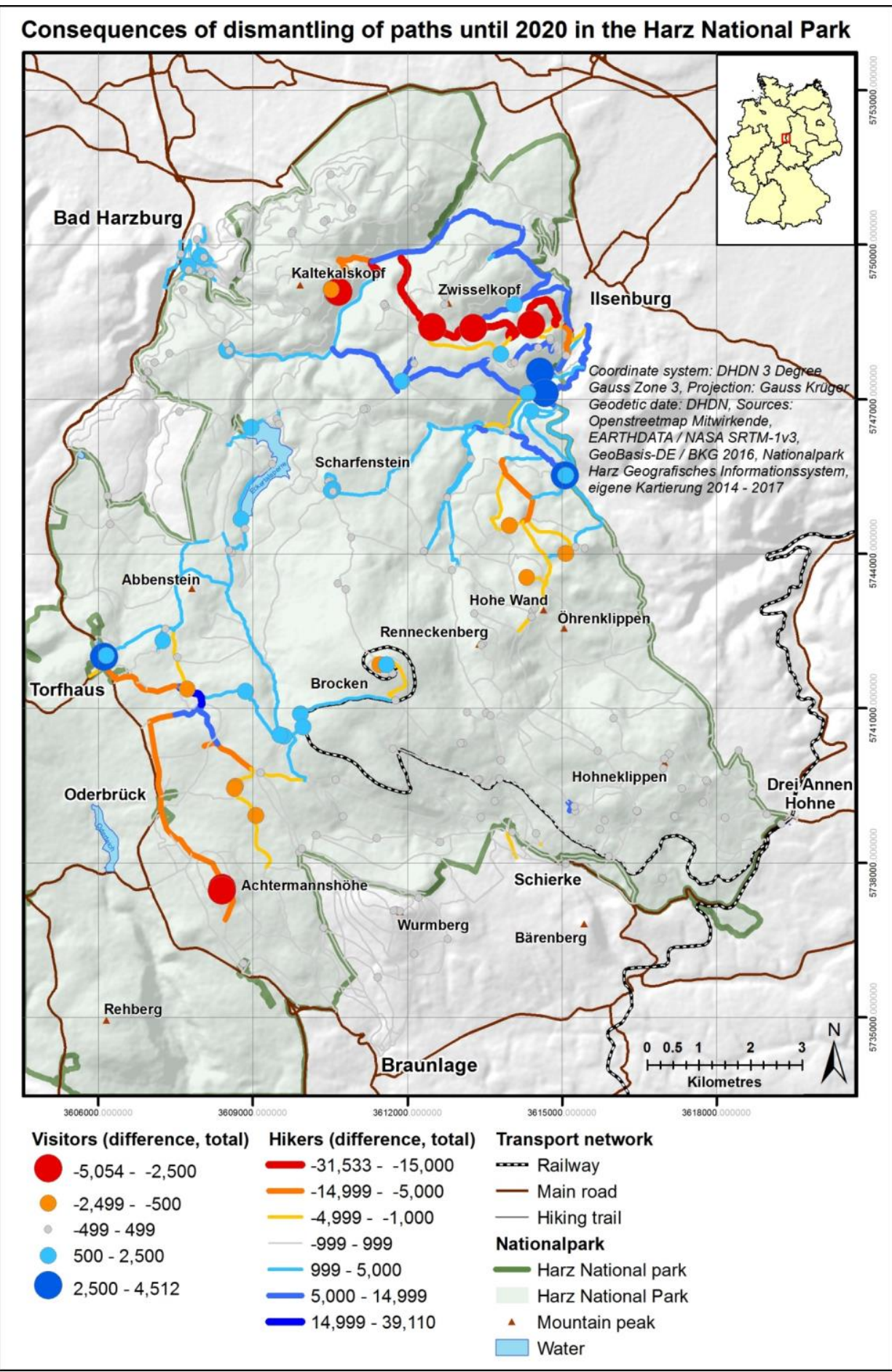

Figure 9: Consequences of dismantling paths up to 2020 in the Harz NP 
The 'Brockenhikers' increasingly also visit POIs along the main routes used. By contrast, in the area around Plessenburg, hardly any 'Brockenhikers' are found. A similar picture emerges in the northern part of the study area, where north of the line Bad Harzburg-ScharfensteinIlsenburg no hikers of this type are encountered.

The simulation of the dismantling of paths 2011-2020 (Nationalpark Harz, 2011a) shows that a change in the path network without a significant change in the overall number of visitors is not possible. While there are 31,533 fewer hikers on the Brocken, the simulation shows up to 39,110 more hikers on other path segments (see Figure 9). Thus, the ongoing changes in some parts of the Harz NP have a clearly measurable impact on the number of hikers and visitors. For the NP management, this means that potential measures should be analysed in advance and the possible changes weighed carefully. During and after the dismantling of paths to the extent that has been planned, the 12 main axes and 7 hotspots that are already known will continue to be recognizable.

\section{Conclusion and outlook}

This research shows that, using an ABM methodology based on available data, a comprehensive spatial simulation of visitor flows in the area around the Brocken can be successfully created and can be used as a basis for further analysis. Input variables are existing visitor counts, the topology of the network of hiking trails and other characteristics of routes, and starting and finishing points. The attributes of the variables influence not only the degrees of attraction of the starting and finishing points for the groups of hikers, but also their choices of route.

After an initial parameterization, a selective sensitivity analysis was carried out for the parameters tour length, number of winter days, and percentage distribution of the groups of hikertypes whose results were included in the calibration. In this way, the error value $R M S E_{\text {param }}=0,3817$ was reduced significantly, to $R M S E_{\text {calib }}=0,1069$, and the model was successfully adapted to the study area. For the final validation of the basic model, visitor numbers were available from other independent studies. Based on the validated base model, $\mathrm{n}=28$ simulation runs were used to analyse key socio-economic figures (visitor numbers) and the behaviour of hikers under changed conditions, thus demonstrating the effects of current management measures to reduce the density of paths. The results of the basic model for the 12 main routes and 7 hotspots identified are in line with the regional level of knowledge of the National Park Administration. This verified comparison of spatially aggregated characteristic values of the model, in addition to the validation, is a strong indication of a consistent basic model.

The model represents a flexible, modular starting point for further modelling and answering in-depth questions of NP Management. The sensitivity analysis was performed on 3 parameters only, however, and should be extended to other parameters in order to create a generic model for different NP and hiking areas. Of particular importance would be parameterization using a terrain model (DEM). In this way, travel speeds could be differentiated and the suitability of the terrain for different groups of hikers and types determined. The carparks are located on the outskirts of the study area, and the model assumes that all vehicle occupants 
are hiking towards the study area. In the surrounding area, however, there are also a large number of potential POIs. Additionally, carparks could be full, which would influence the model, since busy carparks lead to a lower level of attractiveness. These improvements should be the subject of further work.

\section{References}

Abar, S., Theodoropoulos, G. K., Lemarinier, P., \& O'Hare, G. M. (2017). Agent Based Modelling and Simulation tools: A review of the state-of-art software. Computer Science Review. Advance online publication. https://doi.org/10.1016/j.cosrev.2017.03.001

Anhalt, C. O., \& Cortez, R. (2015). Modelling: A structured process. Mathematics Teacher, 108(6).

Banos, A., Lang, C., \& Marilleau, N. (2015). Agent-based spatial simulation with Netlogo. London, Oxford, UK: ISTE Press Ltd; Elsevier Ltd.

Basak, S., Mazumdar, B. D., \& Patheriya, C. B. (2013). Multi-agent paradigm for cognitive parameter based feature similarity for social influence. IJRET: International Journal of Research in Engineering and technology, 2(4), 687-691.

Baumgartner, M. (2013). Agentenbasierte Modellierung von Besucherströmen im Wildnispark Zürich (Masterthesis). Universität Zürich, Zürich.

Europark Deutschland. (2010). Richtlinien für die Anwendung der IUCN-Managementkategorien für Schutzgebiete. Berlin. Retrieved from https://www.bfn.de/fileadmin/MDB/documents/themen/gebietsschutz/IUCN_Kat_Schutzgeb _Richtl_web.pdf

Ferber, J. (1995). Les systèmes multi-agent: vers une intelligence collective. Informatique Intelligence Artificielle.

GAMA Community. (2017). GAMA. Retrieved from http://gama-platform.org/

Grimm, V., Berger, U., DeAngelis, D. L., Polhill, J. G., Giske, J., \& Railsback, S. F. (2010a). The ODD protocol: A review and first update. Ecological Modelling, 221(23), 2760-2768. https://doi.org/10.1016/j.ecolmodel.2010.08.019

Grimm, V., Berger, U., DeAngelis, D. L., Polhill, J. G., Giske, J., \& Railsback, S. F. (2010b). The ODD protocol. A review and first update.: Supplement 2, ODD Template. Retrieved from http://www.ufz.de/export/data/2/100067_ODD_Update_template.doc

Grimm, V., \& Railsback, S. F. (2013). Individual-based Modeling and Ecology: Princeton University Press.

Groß, S., \& Menzel, A. (2016). GPS-Tracking in touristischen Destinationen - Neue SoftwareAnwendung zur Erfassung des Mobilitätsverhaltens am Beispiel von Wanderern im Harz. Raumforschung und Raumordnung, 74(1), 51-68. https://doi.org/10.1007/s13147-016-0381-z

Heckbert, S., Baynes, T., \& Reeson, A. (2010). Agent-based modeling in ecological economics. In K. Limburg \& R. Costanza (Eds.), Annals of the New York Academy of Sciences: Vol. 1185. Ecological economics reviews (pp. 39-53). Boston, Mass.: Blackwell.

Hedderich, J., \& Sachs, L. (2016). Angewandte Statistik: Methodensammlung mit R (15., überarb. und erw. Aufl.). Berlin [u.a.]: Springer Spektrum.

Job, H., Woltering, M., Schamel, J., \& Merlin, C. (2014). Regionalökonomische Effekte des Nationalparks Harz: Endbericht. Würzburg.

Maki, D., \& Thompson, M. (2017). The Mathematical Modling Cycle. Retrieved from http://www.indiana.edu/ hmathmod/modelmodel.html

Menzel, A., Groß, S., \& Drexer, A. (2013, November). Die 'neue' Wanderlust im Har:: Verbalten, Bediurfnisse, Zufriedenheit: Präsentation der Ergebnisse aus der Befragung 'Wandern und GPS im Harz' und des Projektes 'GPS-Tracking von Wanderern im Hař', Wernigerode. 
Nareyek, A. (2001). Constraint based agents: An architecture for constraint-based modeling and local-search-based reasoning for planning and scheduling in open and dynamics worlds. Lecture Notes in Computer Science: 2062. Lecture notes in artificial intelligence. Berlin: Springer.

Nationalpark Harz. (2011a). Nationalparkplan 2011 - 2020. Wernigerode.

Nationalpark Harz. (2011b). Wegeplan 2011 - 2020. Wernigerode.

O'Connor, A., Zerger, A., \& Itami, B. (2005). Geo-temporal tracking and analysis of tourist movement. Mathematics and Computers in Simulation, 69(1), 135-150.

O'Sullivan, D., \& Perry, G. (2013). Spatial Simulation: Exploring Pattern and Process: Wiley-Blackwell.

Prószyńska-Bordas, H. (2017). Visitor segmentation in the National Parks based on motivation and benefits obtained during the stay. Ekonomia i Srodowisko, 2(61), 149-192.

Railsback, S. F., \& Grimm, V. (2012). Agent-based and individual-based modeling: A practical introduction. Princeton, N.J: Princeton University Press.

Rupf, R. (2014). Choice-Experimente als Grundlage für Agenten-basierte Modelle zur Planung im naturorientierten Outdoorsport. Wandern und Mountainbiking in Tourismus- und Bergregionen sowie Schutzgebieten (Dissertation). Universität für Bodenkultur Wien, Wien.

Šalamon, T. (2011). Design of agent-based models: Developing computer simulations for a better understanding of social processes. Academic series. Řepín-Živonín: Tomáš Bruckner.

Schliebitz, A. (2013). Haræ: [mit großer Reisekarte] (10. Aufl., völlig überarb. u. neu gestaltet). Ostfildern: Baedeker.

Steingaß, F., \& Wendt, R. (2006). Bausteine des sozio-ökonomischen Monitorings im Nationalpark Harz. In Biospärenreservat Vessertal-Thüringer Wald (Ed.), Besuchermonitoring und ökonomische Effekte in Nationalen Naturlandschaften (pp. 60-65). Schmiedefeld am Rennsteig.

Wooldridge, M. (2013). Intelligent Agents. In G. Weiss (Ed.), Intelligent robotics and autonomous agents. Multiagent systems (2nd ed., pp. 3-50). Cambridge, Massachusetts, London, England: The MIT Press. 